\title{
WEST Tool Sort
}

The WEST Tool Sort is a self-report evaluation of an individual's perception of his or her ability to perform work tasks using a variety of tools. It is used to explore an individual's attitude toward his or her disability. A secondary use of the WEST Tool Sort is to identify the symptom magnification syndrome, defined by Leonard N. Matheson (1988) as a conscious or unconscious report/display of symptoms used to control the life circumstances of the individual. The WEST Tool Sort is based on the RISC Tool Sort developed by Matheson in 1979. Modifications to the RISC procedure were made by Work Evaluation Systems Technology (WEST) and published as the WEST Tool Sort.

The WEST Tool Sort can be used as an indirect measure of improvement in physical work performance or tool use as the result of a work-hardening program. If an individual's physical work performance improves, perception of work performance should also improve. Therefore, an individual who performs the tool sort before entering and after completing a work-hardening program should demonstrate improvement on the posttest. If the individual's perception of his or her disability is accurate, the WEST Tool Sort can be used to identify areas of limitation. During the evaluation process the examiner can obtain information regarding an individual's job tasks and the tools required for the job. If the individual is not returning to a specific job, the WEST Tool Sort can be used to assist with vocational exploration. This testing instrument can also be used to educate the individual regarding inconsistencies in his or her perceived abilities. Since the WEST Tool Sort relies on self-report and the individual's perception of abilities, the results of the tool sort evaluation should be compared whenever possible with observation of tool use to establish the test's validity.

The WEST Tool Sort is most relevant for the male industrial worker since most of the tools de- picted on the cards are most frequently encountered in an industrial setting. The test consists of 65 cards, each showing a tool being held in a right hand-dominant grasp. The name of the tool is listed on the card in six different languages: English, Spanish, French, German, Vietnamese, and Japanese. A description of the tool's use is provided on the reverse side of the card. Weight and length of the tool and minimum grip strength necessary for use are provided on the reverse side of the card. The individual being tested is asked to sort each of these cards into one of five categories according to his or her perceived ability to use the tool after injury:

1. Category A: I would have no change in the speed at which I work.

2. Category B: I would have a decrease in the speed at which I work.

3. Category C: I would be unable to continue work without an extra break.

4. Category D: I would be unable to work.

5. Category DK: I don't know whether or not I could use this tool.

Three pairs of cards are duplicates of each other and are used to evaluate the consistency of the evaluee's responses. After the evaluee sorts the cards, the examiner records the results of the sort. The examiner then checks for discrepancies in the categories into which the duplicate cards were sorted. If a discrepancy is found, the evaluee is asked to solve it by assigning the duplicate cards to one category. The examiner then performs a "debriefing" session during which the evaluee is asked to justify his or her choice of category for each tool and to describe any symptoms that would limit use of the tool. During the debriefing process, several work function themes (the patient's perception of her or his functional work limitations) are identified. For example, when asked why he or she would have to slow down when 
using a particular tool, the evaluee might respond that the pulling motion required would produce shoulder pain. Tools that have similar functional demands should precipitate similar symptom responses.

The debriefing process is also used to identify the symptom magnification syndrome. The subject is considered to be magnifying symptoms if 1) there are obvious inconsistencies in tool card placement or in the description of symptoms among tools that have similar functional demands, 2) the evaluee is unable to provide a logical rationale for the placement of cards, or 3) during the debriefing session the evaluee frequently moves cards into a category of higher function.

The WEST Tool Sort requires approximately 1 hour to administer and 1 hour to score. The test is accompanied by a 27-page procedure manual and an audio cassette. The procedure manual covers all aspects of test administration and scoring. However, the section on scoring and interpretation could be clearer. The audio cassette clarifies the purpose of the test and the criteria used to identify the symptom magnification syndrome. Objectivity of the WEST Tool Sort could be im- proved if the scoring were quantified and a threshold score for the determination of a diagnosis of symptom magnification were established. According to Matheson (1988) specific rehabilitation approaches can be developed during a workhardening program to address the problem of symptom magnification and enhance the probability of a successful rehabilitation outcome. Therefore, quantitatively and objectively defining the symptom magnification syndrome based on test results would be extremely important both in establishing the diagnosis and justifying its treatment. Furthermore, both reliability and validity studies published in a refereed journal are needed to validate the test.

In summary, the WEST Tool Sort is a testing instrument designed to address the very complex area of a worker's perceptions of function and attitudes toward work. Given the complexity of this type of evaluation, the WEST Tool Sort provides a method for analyzing some aspects of perception and attitudes. Further quantification and research would enhance the practical application of this instrument.

Deborah Lechner, $M S, P T$ Quinn Millington Software Analyst

\section{REFERENCE}

Matheson, L. N. (1988). Symptom magnification syndrome. In Work injury. Aspen, Rockville. 